\title{
Extração de eucalipto com grapple skidder: abordagem de produtividade operacional e custos de produção
}

\author{
Eucalyptus extraction with grapple skidder: operational productivity \\ approach and production costs
}

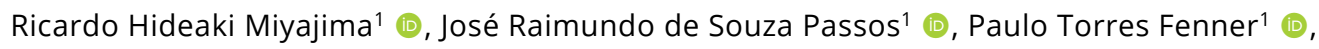 \\ Danilo Simões ${ }^{2}$ (1) \\ ${ }^{1}$ Universidade Estadual Paulista - UNESP, Botucatu, SP, Brasil \\ ${ }^{2}$ Universidade Estadual Paulista - UNESP, Itapeva, SP, Brasil
}

Como citar: Miyajima, R. H., Passos, J. R. S., Fenner, P. T., \& Simões, D. (2020). Extração de eucalipto com grapple skidder: abordagem de produtividade operacional e custos de produção. Scientia Forestalis, $48(128)$, e3298. https://doi.org/10.18671/scifor.v48n128.14

\section{Resumo}

Dentre as atividades da colheita florestal mecanizada, uma das mais importantes é a extração das árvores de eucalipto, comumente realizada por meio do grapple skidder, que é uma máquina intrínseca aos modais de colheita, mais utilizados atualmente no Brasil. Diante disso, aplicou-se a técnica do estudo de tempos e métodos com o objetivo de determinar os custos de produção da operação de um grapple skidder em diferentes condições operacionais. O estudo foi conduzido na região Centro-Oeste do Estado de São Paulo, em que havia uma floresta plantada com Eucalyptus platyphylla. A extração das árvores foi realizada por um grapple skidder da marca John Deere. Foi aplicado o estudo de tempos e métodos com vistas a determinar a produtividade por hora efetiva da máquina, ademais, adotou-se a metodologia preconizada pela Food and Agriculture Organization of the United Nations (FAO, 1992) para estimar o custo por hora programada da máquina. O custo de produção da operação foi obtido pela razão entre o custo por hora programada da máquina e a produtividade efetiva. As análises dos resultados demonstraram que os tempos dos elementos do ciclo operacional com deslocamentos do grapple skidder são os que possuem maior representatividade no tempo total da operação e que a produtividade por hora efetiva da máquina com grapple skidder é influenciada pela distância de extração e pela inclinação da superfície do terreno.
\end{abstract}

Palavras-chave: Colheita de madeira; Eucalyptus; Floresta plantada; Tempos e métodos; Apoio à decisão.

\begin{abstract}
Among the activities of the mechanized forest harvesting, one of the most important is the extraction of eucalyptus trees, commonly carried out by means of the grapple skidder which is an intrinsic machine for harvesting modalities, currently used in Brazil. Therefore, the time and method study technique was applied to determine the production costs of operating a grapple skidder under different operating conditions. The study was conducted in the central western region of São Paulo State, where there was a forest planted with Eucalyptus platyphylla. The tree extraction was carried out by a John Deere grapple skidder. The time and study method was applied to determine the productivity per effective hour of the self-propelled machine, in addition, the methodology by the Food and Agriculture Organization of the United Nations (FAO, 1992) was adopted to estimate the programmed hourly cost of the self-propelled machine. The production cost of the operation was obtained by the ratio between the programmed hourly cost of the machine and the effective productivity. The analysis of the results showed that the times of the operating cycle elements with grapple skidder displacements are those that have greater
\end{abstract}

Fonte de financiamento: Coordenação de Aperfeiçoamento de Pessoal de Nível Superior - Brasil (CAPES) - Código de Financiamento 001.

Conflito de interesse: Nada a declarar.

Autor correspondente: richidetoshimiyajima@hotmail.com

Recebido: 25 fevereiro 2019.

Aceito: 6 novembro 2019.

Editor: Paulo Henrique Müller Silva. (c) (i) Este é um artigo publicado em acesso aberto (Open Access) sob a licença Creative Commons Attribution, que permite uso, distribuição e 
representativeness in the total operation time, and that the productivity per effective hour of the selfpropelled machine with grapple skidder is influenced by the extraction distance and by the slope of the terrain.

Keywords: Wood harvesting; Eucalyptus; Planted forest; Time and method; Decision support.

\section{INTRODUÇÃO}

Atualmente existe uma tendência em adotar máquinas e equipamentos florestais com capacidade operacional que permitam a otimização das operações de colheita de madeira, conseguinte, à minimização dos custos de produção das operações.

Pois o custo com a colheita e transporte representam $50 \%$ ou mais dos dispêndios finais até a madeira posta no pátio da indústria (Moreira et al., 2004; Machado, 2014), devido a sua importância, a busca e o aperfeiçoamento de técnicas que visem melhorias são constantemente essenciais (Minette et al., 2004). Uma das formas para buscar esses avanços é por meio do estudo de tempos e métodos. Este é uma ferramenta que pode ser utilizada com o intuito da busca por progressos, planejamento, controle e racionalização das operações (Fernandes et al., 2009; Simões et al., 2014).

Em meio às atividades na colheita de madeira, a extração é uma das etapas de grande importância e complexidade, pois envolvem fatores como: experiência e habilidade do operador, distância de extração, tipo de solo, declividade, sistema, finalidade da madeira, características do povoamento florestal, capital disponível, desempenho da máquina, entre outros (Tiernan et al., 2004; Seixas \& Castro, 2014; Duka et al., 2018).

Uma das máquinas utilizadas para a realização desta operação é o grapple skidder, encontrado no mercado em diversas marcas, modelos, tamanhos e tipos. Esta é uma máquina florestal, que pode ser articulado com tração $4 \times 4,6 \times 6$ ou $8 \times 8$, desenvolvido para o arraste ou extração da madeira (Lima \& Leite, 2014), o sistema de rodagem pode ser de pneus, semiesteiras ou esteiras (Souza et al., 2002).

Devido à tendência de utilização do grapple skidder na extração de madeira, tornam-se imperativos estudos que contemplem os principais fatores que influenciam a produtividade por hora efetiva da máquina, por consequência, os custos de produção da operação. Nesta perspectiva, Rocha et al. (2009), Hiesl \& Benjamin (2015), Visser \& Stampfer (2015) e Moskalik et al. (2017), descreveram que dentre estes, destacam-se o tempo de experiência dos operadores, a distância de extração, a declividade do terreno, a produtividade de plantios e as espécies florestais.

Diante deste contexto, a análise da produtividade efetiva e dos custos envolvidos na extração de árvores de eucalipto realizada com grapple skidder, considerando os diferentes fatores de interferência, é justificada com vistas à otimização do macroplanejamento das empresas de base florestal. À vista disso, como premissa aplicou-se a técnica do estudo de tempos e métodos com o objetivo de determinar os custos de produção da operação de um grapple skidder em diferentes condições operacionais.

\section{MATERIAL E MÉTODOS}

\section{Área de estudo}

O estudo foi conduzido no Centro-Oeste do Estado de São Paulo, entre as coordenadas geográficas $23^{\circ} 06^{\prime} \mathrm{N} \mathrm{e}, 48^{\circ} 36^{\prime} \mathrm{L}$. De acordo com a classificação de Köppen-Geiger, a região é caracterizada como Cwa - clima tropical de altitude (Alvares et al., 2014). Esta área possui unidades predominantemente do tipo Latossolo Vermelho Amarelo, Distrófico Psamítico (Santos et al., 2018). A superfície do terreno da área experimental foi considerada em classes de declividades conforme a classificação brasileira de solos, logo, com relevo plano de 0 a $3 \%$ (Classe 1) e ondulado de 8 a 20\% (Classe 2), os quais apresentavam acidez forte $(\mathrm{pH}<4,5)$.

Nesta área havia uma floresta plantada com Eucalyptus platyphylla, clonal, com 72 meses de idade, em primeiro corte e espaçamento de $3 \mathrm{~m} \times 2 \mathrm{~m}$. Os indivíduos possuíam altura 
média de 24,58 $\pm 4,78 \mathrm{~m}$, volume médio individual de $0,23 \pm 0,11 \mathrm{~m}^{3}$ e diâmetro à altura do peito (DAP) médio $15,94 \pm 3,85 \mathrm{~cm}$. Desta maneira, os valores foram estimados a partir do inventário florestal, ponderando a amostragem estratificada aleatória. Ademais, as árvores foram derrubadas por um feller-buncher e alocadas nos interiores dos talhões a $90^{\circ} \mathrm{em}$ relação ao alinhamento do plantio com a base dos feixes de madeira direcionados para as estradas florestais.

\section{Coleta dos dados da extração das árvores}

A extração das árvores foi realizada por um grapple skidder da marca John Deere, modelo $848 \mathrm{H}$, articulada, com potência nominal de $200 \mathrm{hp}$, com sistema rodante de pneumáticos, que possuíam banda de rodagem de 35,5 polegadas e roda de 32 polegadas, outrossim, com um grapple skidder do tipo pinça acoplado na parte traseira, com área útil de carga de 1,5 $\mathrm{m}^{2}$, consentindo o acúmulo de dois feixes de madeira dispostos pelo feller-buncher, isto é, em média 22 árvores por ciclo operacional. O ramal de extração dos feixes de madeira era composto por dois eitos de derrubada do feller-buncher, por consequência, seis linhas de plantio.

O ciclo operacional do grapple skidder foi composto por elementos que resultaram em produção, ou seja, considerou-se somente o tempo efetivo da operação, compostos por: deslocamento sem carga; deslocamento com carga, carregamento da madeira; e descarregamento da madeira. Portanto, o deslocamento sem carga era caracterizado do momento em que o grapple skidder fecha a garra e inicia o deslocamento em direção ao interior do talhão até o momento que a máquina inicia abertura da garra para a carga do primeiro feixe. O carregamento com carga inicia com a abertura da garra para carga do primeiro feixe até o momento em que a máquina fecha a garra após carga do último feixe e inicia o deslocamento até a beira do talhão.

O deslocamento com carga ocorreu do momento em que a máquina fecha a garra após carga do ultimo feixe e inicia o deslocamento até a beira do talhão até o momento em que o grapple skidder já na beira do talhão, inicia abertura da garra para formar o estaleiro. O descarregamento da madeira ocorreu do momento em que a máquina já na beira do talhão inicia abertura da garra para formar o estaleiro até o momento em que o grapple skidder fecha a garra vazia e inicia o deslocamento em direção ao interior do talhão.

\section{Análise técnica}

Com o intuito de avaliar a operação executada pela máquina, foi realizado o estudo de tempos e métodos, por meio do método de tempo contínuo (Magagnotti \& Spinelli 2012; Simões et al., 2018). A suficiência amostral foi determinada a partir da cronometragem de uma amostra realizada preliminarmente, portanto, foram observados 615 ciclos operacionais com o propósito de obter uma amostra com validade estatística, pautada nos tempos médios dos elementos do ciclo de trabalho $(\bar{x})$ e no desvio padrão da amostra $(s)$, com nível de confiança de 95\% e erro de 5\%, descrita na Equação 1 em consonância a Stevenson (2001).

$$
n=\left(\frac{z s}{a \bar{x}}\right)^{2}
$$

em que:

\footnotetext{
$n$ é o tamanho da amostra;

$z$ é o número de desvios padrões em uma distribuição normal necessários para se obter;

$s$ é o desvio padrão da amostra;

$a$ é o percentual de precisão desejado; e

$\bar{x}$ é a média da amostra.

A produtividade por hora efetiva da máquina foi determinada pela razão entre o volume de madeira extraído e a quantidade de horas efetivas de operação (Equação 2).
} 
$P H M=\frac{V_{\text {ext }}}{h}$

em que:

PHM é a produtividade por hora efetiva da máquina $\left(\mathrm{m}^{3} \mathrm{~h}^{-1}\right)$;

$V_{\text {ext }}$ é o volume de madeira extraído $\left(\mathrm{m}^{3}\right)$; e

$h$ é a quantidade de horas efetivas da operação (h).

\section{Delineamento experimental}

O estudo avaliou fatores que comumente influenciam na produtividade operacional e, por conseguinte, nos custos de produção, conforme Leonello et al. (2012). Portanto, foram considerados dois operadores com tempos distintos na operação, caracterizados como: Operador 1, o qual possuía 24 meses; e Operador 2 que tinha 29 meses de experiência profissional. Ademais, foram consideradas as duas classes de declividade (Classe 1 e a Classe 2), e ainda, quatro distâncias de extração, as quais compreenderam 50, 100, 150 e 200 metros.

Para os elementos do ciclo de trabalho, da produtividade por hora efetiva da máquina e do custo de produção da operação, considerando os fatores classes de declividades, distância de extração e operador em uma estrutura fatorial, foram aplicados modelos lineares generalizados com distribuição de probabilidade gama e função de ligação logarítmica (Nelder \& Wedderburn, 1972; Diggle et al., 2002), pois conforme o teste de Shapiro-Wilk os valores amostrais rejeitaram a hipótese de normalidade dos dados $(p<0,05)$.

Para comparações entre tratamentos foi utilizado o teste de Tukey-Kramer do comando LS Means do procedimento Genmod do programa Statistical Analysis System (SAS Institute, 2012). Além disso, ajustaram-se modelos de regressão linear para as variáveis dependentes: produtividade por hora efetiva da máquina e custo de produção da operação com a máquina, considerando as variáveis independentes distância de extração, classe de declividade e operador, como variáveis dummy, por meio do procedimento GLM do SAS.

\section{Análise econômica}

Os valores monetários foram expressos em dólar comercial americano (US\$), assim, foi considerada a taxa de câmbio da divisa estrangeira medido em unidades e frações da moeda nacional, que era de $\mathrm{R} \$ 3,7135$ em 11 de janeiro de 2019, conforme o Banco Central do Brasil (2019).

O custo por hora programada da máquina $\left(C_{h p}\right)$ foi determinado de acordo com a metodologia de controle de custos na colheita florestal mecanizada preconizada pela Food and Agriculture Organization of the United Nations (1992), classificados em custos fixos de depreciação, remuneração do capital, seguro, taxas sobre propriedade, mão de obra, e em custos variáveis de combustível, óleos lubrificantes e graxas, manutenção e reparos e, peças de reposição.

A vida econômica do grapple skidder foi estimada em cinco anos, com valor de revenda de $20 \%$ do preço de aquisição. Além disso, os encargos sociais corresponderam a $134 \%$ sobre o salário do operador da máquina autopropelida. A remuneração do capital decorrente do uso do dinheiro investido para a aquisição da máquina foi calculado por meio do Custo Médio Ponderado de Capital (CMPC) conforme descrito na Equação 3, devido à empresa de base florestal possuir passivo oneroso.

$C M P C=k_{b}(1-I R) \frac{B}{B+S}+k_{s} \frac{S}{B+S}$

em que:

$k_{b}$ é o custo de capital de terceiros; 
IR é alíquota de imposto sobre a renda;

$B$ é o valor presente da riqueza dos detentores;

$S$ é o valor presente da riqueza dos acionistas; e

$k_{s}$ é o custo de capital dos acionistas.

Assim, empregou-se o Modelo de Precificação de Ativos (CAPM), com a finalidade de determinar o custo do capital dos acionistas, considerando o risco sistemático de empresas do mercado acionário listadas na B3BOVESPA do setor de madeira e papel (Equação 4).

$K=R_{F}+\beta\left(R_{M}-R_{F}\right)+\alpha_{B R}$

em que:

$K$ é a taxa de desconto ajustada ao risco;

$R_{F}$ é a taxa de juros livre de risco (Annual Return on Treasury bonds 10Y US);

$\beta$ é o coeficiente sistemático da indústria de madeira e papel;

$R_{M}$ é o retorno da carteira de mercado (S\&P Global Timber \& Forestry Index);

$\left(R_{M}-R_{F}\right)$ é o prêmio pelo risco; e

$\alpha_{B R}$ é o prêmio de risco do país.

O custo de produção da operação, determinante tanto na definição do modal de colheita, quanto na rentabilidade dos produtos das empresas de base florestal, foi calculado pela razão entre o custo por hora programada do grapple skidder e a produtividade por hora efetiva da máquina (Equação 5).

$C_{p m}=\frac{C_{h p}}{P_{h m}}$

em que:

$C_{p m}$ é o custo de produção da operação com a máquina (US\$ $\mathrm{m}^{-3}$ );

$C_{h p}$ é custo por hora programada da máquina $\left(U S \$ \mathrm{~h}^{-1}\right)$.

\section{RESULTADOS E DISCUSSÃO}

\section{Tempos do ciclo operacional}

Ao sopesar a suficiência amostral na Classe 1, foram observados 76 e 180 ciclos operacionais respectivamente para o Operador 1 e para o Operador 2, o que permitiu extrair $1.438,75 \mathrm{~m}^{3}$ de madeira. Já para a Classe 2 foram monitorados 203 e 156 ciclos operacionais concomitantemente para o Operador 1 e para o Operador 2 , o que resultou em 1.325,06 $\mathrm{m}^{3}$ de madeira extraída. Neste sentido, o tamanho da amostra foi superior à quantidade de ciclos mínimos a serem observados com legitimidade estatística.

Durante o período do estudo na Classe 1, o tempo efetivo de trabalho para o Operador 1 foi 4 horas e 50 minutos e 10 horas e 18 minutos para Operador 2. Na Tabela 1, observouse que os elementos deslocar sem carga e deslocar com carga foram os que demandaram maiores tempos para serem executados, ou seja, representou um percentual médio de $79,01 \%$ do total da operação, porém apenas para a distância de extração de $200 \mathrm{~m}$ para o elemento deslocar com carga foi estatisticamente significativo $(p<0,05)$ entre os operadores.

Não obstante, ao sopesar as distâncias de extração, constatou-se diferença estatística $(p<0,05)$ entre os tempos destes elementos, isto pode indicar que ocorreu um aumento do tempo de deslocamento, como previsto, sobretudo, corroborado por Pereira et al. (2015), os quais destacam que esses elementos são influenciados pelo comprimento do ramal de 
deslocamento da máquina, e também por fatores como declividade do terreno, tamanho dos talhões, condições da trilha de arraste e presença de obstáculos.

Ao analisar os elementos carregamento e descarregamento da madeira, verificou-se, que foram as atividades as quais demandaram menor tempo para a execução, por outra forma, representaram 20,99\% do total da operação, condição análoga constatada por Proto et al. (2018). Na análise do carregamento, entre os operadores, foi possível afirmar que ocorreram diferenças estatísticas significativas $(p<0,05)$ somente para a distância de $100 \mathrm{~m}$, já para o descarregamento, este fato foi constatado para todas as distâncias de extração.

Diante disso, $62,5 \%$ dos tempos analisados destes elementos, apresentaram diferenças estatísticas significativas entre os operadores, portanto, pode-se considerar que o tempo de experiência profissional para esta condição exerceu influência para a realização destes elementos, outro fato que pode explicar essas diferenças deve-se pela má formação dos feixes, sendo este o único fator não considerado no estudo, inclusive, Lopes et al. (2009) e Hiesl et al. (2015) relatam que uma das explicações para o maior consumo de tempo para a realização do elemento carga da madeira é atribuída pela disposição das madeiras dos feixes a serem carregados.

Tabela 1: Tempo médio dos elementos (minutos/ciclo) e coeficiente de variação (\%) da extração das árvores realizada por um grapple skidder para a Classe 1.

\begin{tabular}{ccccccccc}
\hline \multirow{2}{*}{ Distâncias de extração } & \multicolumn{2}{c}{ DSC } & \multicolumn{2}{c}{ DCC } & \multicolumn{2}{c}{ CM } & \multicolumn{2}{c}{ DM } \\
\cline { 2 - 9 } & Op. 1 & Op. 2 & Op. 1 & Op. 2 & Op. 1 & Op. 2 & Op. 1 & Op. 2 \\
\hline \multirow{3}{*}{$50 \mathrm{~m}$} & $0,68 \mathrm{Aa}$ & $0,76 \mathrm{Aa}$ & $0,73 \mathrm{Aa}$ & $0,68 \mathrm{Aa}$ & $0,38 \mathrm{Aa}$ & $0,32 \mathrm{Aa}$ & $0,24 \mathrm{Aa}$ & $0,12 \mathrm{Ba}$ \\
& $(43,60)$ & $(51,77)$ & $(47,81)$ & $(58,31)$ & $(70,97)$ & $(62,37)$ & $(71,01)$ & $(75,15)$ \\
\multirow{2}{*}{$100 \mathrm{~m}$} & $1,15 \mathrm{Ab}$ & $1,32 \mathrm{Ab}$ & $1,20 \mathrm{Ab}$ & $1,33 \mathrm{Ab}$ & $0,59 \mathrm{Ab}$ & $0,38 \mathrm{Bb}$ & $0,21 \mathrm{Aa}$ & $0,12 \mathrm{Ba}$ \\
& $(29,42)$ & $(28,67)$ & $(33,30)$ & $(31,84)$ & $(56,11)$ & $(65,46)$ & $(73,31)$ & $(67,53)$ \\
\multirow{3}{*}{$150 \mathrm{~m}$} & $1,67 \mathrm{Ac}$ & $1,52 \mathrm{Ac}$ & $1,75 \mathrm{Ac}$ & $1,99 \mathrm{Ac}$ & $0,72 \mathrm{Ac}$ & $0,76 \mathrm{Ac}$ & $0,22 \mathrm{Aa}$ & $0,12 \mathrm{Ba}$ \\
& $(31,38)$ & $(23,91)$ & $(24,99)$ & $(27,65)$ & $(42,79)$ & $(51,27)$ & $(65,74)$ & $(41,89)$ \\
\multirow{2}{*}{$200 \mathrm{~m}$} & $2,05 \mathrm{Ad}$ & $1,97 \mathrm{Ad}$ & $2,28 \mathrm{Ad}$ & $1,98 \mathrm{Bd}$ & $0,79 \mathrm{Ad}$ & $0,74 \mathrm{Ad}$ & $0,21 \mathrm{Aa}$ & $0,13 \mathrm{Ba}$ \\
& $(16,01)$ & $(18,62)$ & $(14,88)$ & $(19,02)$ & $(38,48)$ & $(37,18)$ & $(79,76)$ & $(62,53)$ \\
\hline
\end{tabular}

Legenda: DSC: deslocar sem carga; DCC: deslocar com carga; CM: carga da madeira; DM: descarga da madeira; Op. 1: Operador 1; Op. 2: Operador 2. Médias seguidas pela mesma letra maiúscula na linha e médias seguidas da mesma letra minúscula na coluna, não diferem estatisticamente, entre si, pelo teste de Tukey-Kramer com 5\% de significância; valores do coeficiente de variação entre parênteses.

O estudo de tempos e métodos é considerado uma das diretrizes para a maximização dos resultados de uma empresa de base florestal, especificamente para as operações de colheita de madeira, as quais exercem influência direta sobre o preço final dos produtos. Deste modo, o tempo efetivo observado na operação para a Classe 2 foi de 10 horas e 25 minutos para o Operador 1 e 8 horas e 55 minutos para o Operador 2. As atividades deslocamento sem carga e com carga dispenderam $76,88 \%$ do total da operação, sendo $2,13 \%$ inferior à Classe 1. Ademais, os tempos destes elementos para as distâncias de extração de 100 e $200 \mathrm{~m}$ apresentaram diferenças estatisticamente significativas $(p<0,05)$ entre os operadores, as quais podem ser atribuídas ao tempo de experiência profissional.

Ao analisar o deslocamento sem carga (50,100 e $150 \mathrm{~m}$ ) e para o deslocamento com carga (50, 150 e $200 \mathrm{~m}$ ), estas atividades não foram significativas entre os operadores (Tabela 2). Quando analisada a distância de extração, constatou-se diferenças estatisticamente significativas que o aumento desta exerceu influência direta nestes dois elementos avaliados, condição corroborada por Behjou et al. (2008) e Ghaffariyan et al. (2012) os quais enfatizam que o aumento da distância de extração contribui para o aumento do tempo efetivo do ciclo operacional da máquina. 
Quanto ao carregamento da madeira, não foi constatada diferença significativa em nenhuma distância de extração entre os operadores. Já para o descarregamento da madeira foram averiguadas diferenças estatisticamente significativas $(p<0,05)$ para as distâncias 50 , 100 e 150 m. Estas podem ser explicadas pela má formação dos feixes de madeira, e ainda de acordo com Minette et al. (2008), outros fatores que podem interferir no tempo para a realização destes elementos são a habilidade do operador e a quantidade de árvores a ser transportada.

Ao analisar o deslocamento sem carga e com carga da máquina, foi constatado que o tempo médio para a realização destes elementos para o Operador 1 foi 3,97\% e de 3,15\%, ambos os valores foram inferiores ao Operador 2 para a Classe 1 e Classe 2, respectivamente. No entanto, para ambos os operadores e declividades foi averiguado que a máquina consome a maior parte do seu do ciclo operacional com o deslocamento com carga e deslocamento sem carga, fato confirmado por Seixas (2008), isto é, o grapple skidder, consome a maior parte do tempo de operação na execução destas duas atividades.

Tabela 2: Tempo médio dos elementos (minutos/ciclo) e coeficiente de variação (\%) da extração das árvores realizada por um grapple skidder para a Classe 2.

\begin{tabular}{|c|c|c|c|c|c|c|c|c|}
\hline \multirow{2}{*}{ Distâncias de extração } & \multicolumn{2}{|c|}{ DSC } & \multicolumn{2}{|c|}{ DCC } & \multicolumn{2}{|c|}{$\mathrm{CM}$} & \multicolumn{2}{|c|}{ DM } \\
\hline & Op. 1 & Op. 2 & Op. 1 & Op. 2 & Op. 1 & Op. 2 & Op. 1 & Op. 2 \\
\hline $50 \mathrm{~m}$ & $\begin{array}{l}0,70 \mathrm{Aa} \\
(53,29)\end{array}$ & $\begin{array}{l}0,71 \mathrm{Aa} \\
(73,45)\end{array}$ & $\begin{array}{l}0,67 \mathrm{Aa} \\
(80,07)\end{array}$ & $\begin{array}{l}0,62 \mathrm{Aa} \\
(91,73)\end{array}$ & $\begin{array}{l}0,38 \mathrm{Aa} \\
(83,91)\end{array}$ & $\begin{array}{l}0,35 \mathrm{Aa} \\
(84,09)\end{array}$ & $\begin{array}{l}0,27 \mathrm{Aa} \\
(61,15)\end{array}$ & $\begin{array}{l}0,13 \mathrm{Ba} \\
(67,88)\end{array}$ \\
\hline $100 \mathrm{~m}$ & $\begin{array}{l}1,02 \mathrm{Ab} \\
(35,77)\end{array}$ & $\begin{array}{l}1,07 \mathrm{Ab} \\
(43,50)\end{array}$ & $\begin{array}{l}1,43 \mathrm{Ab} \\
(48,32)\end{array}$ & $\begin{array}{l}1,03 \mathrm{Bb} \\
(37,75)\end{array}$ & $\begin{array}{l}0,49 \mathrm{Ab} \\
(80,18)\end{array}$ & $\begin{array}{l}0,49 \mathrm{Aa} \\
(78,72)\end{array}$ & $\begin{array}{l}0,30 \mathrm{Aa} \\
(43,28)\end{array}$ & $\begin{array}{l}0,18 \mathrm{Bb} \\
(82,08)\end{array}$ \\
\hline $150 \mathrm{~m}$ & $\begin{array}{l}1,42 \mathrm{AC} \\
(29,41)\end{array}$ & $\begin{array}{l}1,29 \mathrm{AC} \\
(35,99)\end{array}$ & $\begin{array}{l}1,78 \mathrm{Ac} \\
(31,42)\end{array}$ & $\begin{array}{l}1,72 \mathrm{AC} \\
(31,59)\end{array}$ & $\begin{array}{l}0,55 \mathrm{Ac} \\
(81,77)\end{array}$ & $\begin{array}{l}0,49 \mathrm{Aa} \\
(71,61)\end{array}$ & $\begin{array}{l}0,32 \mathrm{Aa} \\
(55,77)\end{array}$ & $\begin{array}{l}0,20 \mathrm{Bc} \\
(79,46)\end{array}$ \\
\hline $200 \mathrm{~m}$ & $\begin{array}{l}1,75 \mathrm{Ad} \\
(19,41)\end{array}$ & $\begin{array}{l}1,38 \mathrm{Bd} \\
(44,84)\end{array}$ & $\begin{array}{c}2,06 \mathrm{Ad} \\
(19,17)\end{array}$ & $\begin{array}{l}2,29 \mathrm{Ad} \\
(34,92)\end{array}$ & $\begin{array}{l}0,53 \mathrm{Ad} \\
(80,78)\end{array}$ & $\begin{array}{l}0,53 \mathrm{Aa} \\
(71,46)\end{array}$ & $\begin{array}{l}0,25 \mathrm{Aa} \\
(74,13)\end{array}$ & $\begin{array}{l}0,29 \mathrm{Ad} \\
(58,44)\end{array}$ \\
\hline
\end{tabular}

Médias seguidas pela mesma letra maiúscula na linha e médias seguidas da mesma letra minúscula na coluna, não diferem estatisticamente, entre si, pelo teste de Tukey-Kramer com 5\% de significância; valores do coeficiente de variação entre parênteses.

\section{Produtividade por hora efetiva da máquina}

O conhecimento da produtividade por hora efetiva da máquina é de importância para o gestor com vistas, sobretudo, ao macroplanejamento florestal. Desta maneira, ao analisar a produtividade do grapple skidder na Classe 1, foi possível averiguar que o tempo de experiência profissional entre o Operador 1 e Operador 2 apresentou diferença significativa $(p<0,05)$ para as distâncias de extração de 100 e 150 m. Ademais, para a Classe 2, também constatou-se o mesmo fato, entre o Operador 1 e Operador 2 , ou seja, foi estatisticamente significativo $(p<0,05)$ apenas para a distância de extração de $50 \mathrm{~m}$ (Tabela 3).

Não obstante, em outras distâncias de extração (50 e 200 m para a Classe 1) e (100, 150 e $200 \mathrm{~m}$ para a Classe 2) não foram constatadas diferenças significativas da produtividade do grapple skidder entre o Operador 1 e Operador 2. Embora, não tenha apresentando diferença estatística significativa foi verificada em determinadas distâncias de extração que a produtividade por hora efetiva apresentou valores (metros cúbicos de madeira) distintos, os quais ao término de uma jornada diária de trabalho podem influenciar proporcionalmente na produção final da operação, o que corrobora que o tempo de experiência profissional pode influenciar na produtividade por hora efetiva da máquina. Ovaskainen et al. (2004) e Spinelli et al. (2007) complementam que o operador e a sua respectiva habilidade são fatores que exercem influência sob a produtividade da máquina.

Ao ponderar a produtividade por hora efetiva do grapple skidder para o Operador 1 tanto na Classe (1) quanto na Classe (2), apresentaram diferenças estatisticamente significativas 
$(p<0,05)$ para todas as distâncias de extração avaliadas. Já para o Operador 2 para esta condição os valores diferiram somente nas classes 150 e 200 m. Considerando a produtividade da máquina nas classes de declividade, os resultados indicaram que com o aumento do grau de inclinação do relevo houve uma relação inversa, isto é, houve uma diminuição da produtividade sendo diferentes entre si $(p<0,05)$. Ressalta-se ainda que as maiores produtividades efetivas foram obtidas na Classe 1 para ambos os operadores, confirmando os estudos realizados por Oliveira et al. (2008) para um clambunk skidder, Vusic et al. (2013) e por Lopes \& Diniz (2015), para a operação realizada por um skidder de cabo.

Ao analisar a influência da distância de extração na produtividade por hora efetiva da máquina autopropelida nas classes de declividades e entre os operadores, averiguou-se valores que diferem estatisticamente $(p<0,05)$. Ao mesmo tempo, com o aumento da distância de extração houve uma redução na produtividade do grapple skidder (Tabela 3), condição semelhante aos estudos realizados por Birro et al. (2002), Mousavi (2012) e Lopes et al. (2014).

Considerando a distância de extração de $200 \mathrm{~m}$ na Classe 1 a produtividade do grapple skidder para o Operador 1 foi $77,79 \mathrm{~m}^{3} \mathrm{~h}^{-1}$ e para o Operador $286,79 \mathrm{~m}^{3} \mathrm{~h}^{-1}$, valores superiores aos estudos realizados por Fiedler et al. (2008) durante a análise de um skidder em condições de relevo semelhante, isto é, com declividade plana, obtiveram uma produtividade efetiva média de $42,31 \mathrm{~m}^{3} \mathrm{~h}^{-1}$ para uma distância média de 230 metros.

Tabela 3: Produtividade média por hora efetiva da máquina $\left(\mathrm{m}^{3} \mathrm{~h}^{-1}\right)$ e coeficiente de variação (\%) da extração das árvores realizada por um grappe skidder segundo a distância de extração, classe de declividade e operador.

\begin{tabular}{|c|c|c|c|}
\hline Distâncias de extração & Classe & Op. 1 & Op. 2 \\
\hline \multirow{2}{*}{$50 \mathrm{~m}$} & 1 & $\begin{array}{c}163,89 \text { i b C } \\
(30,39)\end{array}$ & $\begin{array}{c}151,96 \text { i a } B \\
(45,01)\end{array}$ \\
\hline & 2 & $\begin{array}{c}111,42 \text { i a C } \\
(58,28)\end{array}$ & $\begin{array}{c}145,60 \text { ā a C } \\
(65,05)\end{array}$ \\
\hline \multirow{2}{*}{$100 \mathrm{~m}$} & 1 & $\begin{array}{c}124,78 \text { à b B } \\
(24,46)\end{array}$ & $\begin{array}{c}94,49 \text { i a } A \\
(27,42)\end{array}$ \\
\hline & 2 & $\begin{array}{c}80,63 \text { i a B } \\
(36,73)\end{array}$ & $\begin{array}{c}88,75 \text { i a B } \\
(50,96)\end{array}$ \\
\hline \multirow{2}{*}{$150 \mathrm{~m}$} & 1 & $\begin{array}{c}101,74 \text { ā b A B } \\
(18,49)\end{array}$ & $\begin{array}{c}89,21 \text { i b A } \\
(16,77)\end{array}$ \\
\hline & 2 & $\begin{array}{c}69,20 \text { i a A B } \\
(27,75)\end{array}$ & $\begin{array}{c}62,42 \text { i a A } \\
(35,42)\end{array}$ \\
\hline \multirow{2}{*}{$200 \mathrm{~m}$} & 1 & $\begin{array}{c}77,79 \text { i b A } \\
(12,37)\end{array}$ & $\begin{array}{c}86,79 \text { i b A } \\
(16,69)\end{array}$ \\
\hline & 2 & $\begin{array}{c}61,95 \text { i a A } \\
(35,42)\end{array}$ & $\begin{array}{c}57,47 \text { i a } A \\
(40,14)\end{array}$ \\
\hline
\end{tabular}

Letras gregas comparam os operadores, segundo o teste de Tukey-Kramer com 5\% de significância. Letras minúsculas comparam as classes, segundo o teste de Tukey-Kramer com 5\% de significância. Letras maiúsculas comparam as distâncias de extração, segundo o teste de Tukey-Kramer com 5\% de significância.

A produtividade por hora efetiva da máquina foi modelada em função das variáveis distâncias de extração, classes de declividade e operador (Equação 6). Ressalta-se que a variável operador, para as distâncias 150 e $200 \mathrm{~m}$ e a Classe 2 não foram significativas $(p<0,01)$ para o modelo ajustado $\left(R^{2}=25,98 \%\right)$, indicando que a distância de extração $50 \mathrm{~m}$ exerceu maior influência positiva na produtividade do grapple skidder, isto posto, a equação resultante da regressão linear foi expressa por:

PHM $=59,40+64,54 d_{50}+21,36 d_{100}+26,94 c_{1}$ 
em que:

$d_{50}$ é a distância de extração $50 \mathrm{~m}$;

$d_{100}$ é a distância de extração 100; e

$c_{l}$ é a Classe 1.

\section{Previsão dos custos}

Em relação à taxa necessária para a remuneração do capital calculada por meio do CMPC, uma das premissas utilizadas foi a taxa de juros livre de risco de $2,36 \%$. O coeficiente sistemático do ativo foi calculado a partir do beta médio total, que resultou em um beta desalavancado médio de 0,33, sucessivamente em um beta realavancado de 0,42. Considerou-se ainda o retorno anualizado da carteira de mercado que foi 5,06\%. E por fim, o prêmio de risco do país foi de 2,37\% calculado por meio da média geométrica do Emerging Markets Bond Index - EMBI+Br. Por conseguinte, obteve-se o CAPM de 6,85\%. Ao sopesar o custo de capital de terceiros de 5,96\% e a proporção do ativo financiado por dívida de $40,84 \%$, o CMPC foi de $5,66 \%$.

À vista disso, o custo por hora programada para o grapple skidder foi de 77,85 US $\$ \mathrm{~h}^{-1}$. Dentre os elementos, o combustível, mão de obra e depreciação foram os que mais oneraram, juntos representam $71,51 \%$ do custo total da máquina (Figura 1). Nesta perspectiva, Santos et al. (2013) e Bodaghi et al. (2018) também adotaram a metodologia da Food and Agriculture Organization of the United Nations para estimar o custo por hora programada de máquinas com características técnicas semelhantes, contudo, obtiveram valores distintos. Estas diferenças podem ser atribuídas principalmente à fatores como tempo e a taxa utilizada para a depreciação da máquina, valores monetários despendidos com mão de obra e os respectivos encargos sociais, os quais são inerentes à cada empresa de base florestal, e ainda, pelas despesas com combustíveis que variam em decorrência de regiões ou de países.

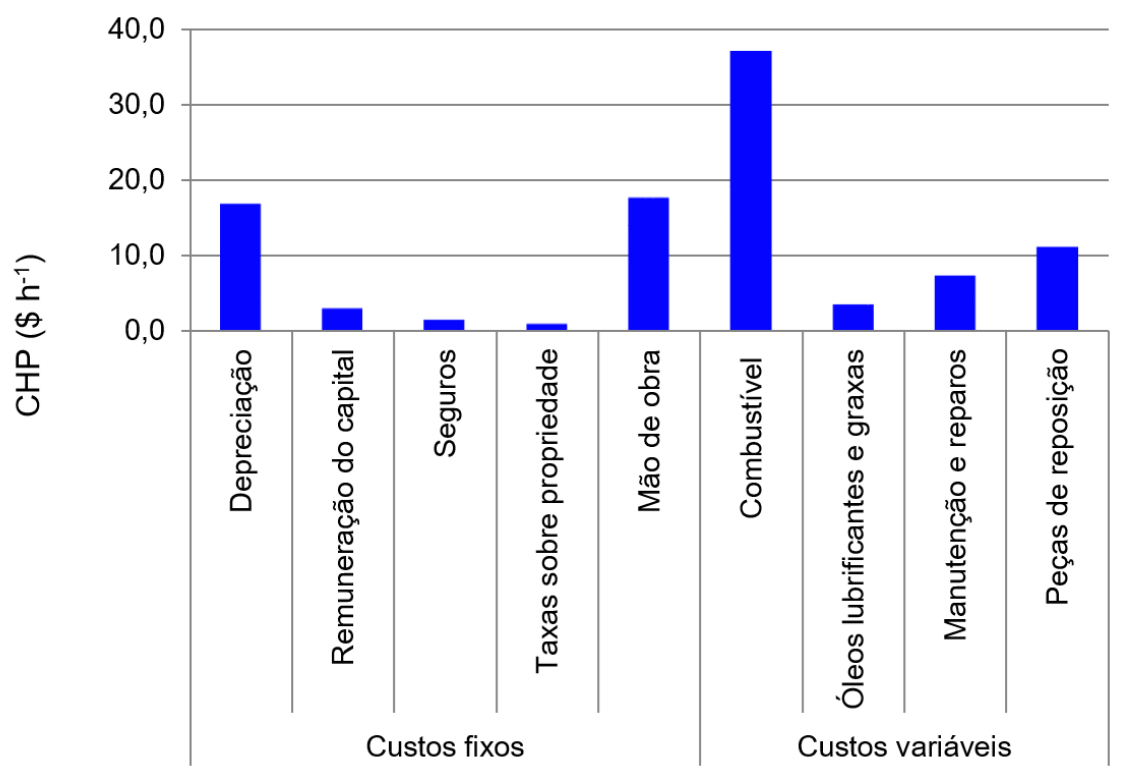

Figura 1: Elementos do custo por hora programada da máquina

Observa-se na Tabela 4 que houve um aumento dos custos de produção da operação conforme o acréscimo da extensão do ramal de extração das árvores, todavia, não foram estatisticamente significativos $(p<0,05)$ em algumas condições operacionais, o que pode ser explicado em decorrência da inconstância entre os valores monetários, aliás, do coeficiente médio de variação que foi $35,81 \%$. Ao considerar os operadores, foi possível aferir, que o custo 
de produção apresentou diferença significativa, indicando que para as condições analisadas o tempo de experiência não exerceu influência sobre estes custos.

Ao analisar o efeito da declividade sobre o custo de produção da operação, foi constatado diferenças estatisticamente significativas $(p<0,05)$ para $75 \%$ das condições operacionais analisadas, uma vez que, existe uma relação negativa entre a declividade do relevo e a produtividade por hora efetiva da máquina autopropelida, conforme discorrido por Diniz et al. (2018).

Tabela 4: Custo médio de produção da operação (US\$ $\mathrm{m}^{-3}$ ) e coeficiente de variação (\%) da extração das árvores realizada por um grapple skidder segundo a distância de extração, classe de declividade e operador.

\begin{tabular}{|c|c|c|c|}
\hline Distâncias de extração & Classe & Op. 1 & Op. 2 \\
\hline \multirow{2}{*}{$50 \mathrm{~m}$} & 1 & $\begin{array}{c}0,51 \text { i a A } \\
(27,29)\end{array}$ & $\begin{array}{c}0,60 \text { i a A } \\
(37,70)\end{array}$ \\
\hline & 2 & $\begin{array}{c}0,99 \text { i b A } \\
(74,13)\end{array}$ & $\begin{array}{l}0,94 \text { i b A } \\
(106,36)\end{array}$ \\
\hline \multirow{2}{*}{$100 \mathrm{~m}$} & 1 & $\begin{array}{c}0,66 \text { i a A B } \\
(23,76)\end{array}$ & $\begin{array}{c}0,88 \text { i a B C D } \\
(26,74)\end{array}$ \\
\hline & 2 & $\begin{array}{c}1,11 \text { i b A B } \\
(42,28)\end{array}$ & $\begin{array}{c}1,02 \text { i a A } \\
(36,21)\end{array}$ \\
\hline \multirow{2}{*}{$150 \mathrm{~m}$} & 1 & $\begin{array}{c}0,79 \text { ¡̇ a B C } \\
(16,88)\end{array}$ & $\begin{array}{c}0,90 \text { i a C D } \\
(22,35)\end{array}$ \\
\hline & 2 & $\begin{array}{c}1,21 \text { i b B C } \\
(27,93)\end{array}$ & $\begin{array}{c}1,38 \text { i b B C } \\
(30,91)\end{array}$ \\
\hline \multirow{2}{*}{$200 \mathrm{~m}$} & 1 & $\begin{array}{c}1,02 \text { i a C } \\
(13,11)\end{array}$ & $\begin{array}{c}0,92 \text { i a D } \\
(18,13)\end{array}$ \\
\hline & 2 & $\begin{array}{c}1,38 \text { i a C } \\
(27,55)\end{array}$ & $\begin{array}{c}1,58 \text { i b c } \\
(41,56)\end{array}$ \\
\hline
\end{tabular}

Letras gregas comparam os operadores, segundo o teste de Tukey-Kramer com 5\% de significância. Letras minúsculas comparam as classes, segundo o teste de Tukey-Kramer com 5\% de significância. Letras maiúsculas comparam as distâncias de extração, segundo o teste de Tukey-Kramer com 5\% de significância.

Os resultados da modelagem do custo de produção da operação em função das variáveis ponderadas no estudo, mostraram que a variável operador, nas distâncias de 150 e $200 \mathrm{~m}$ e a Classe 2 não foram significativas $(p>0,01)$ para a equação de regressão ajustada $\left(R^{2}=\right.$ 19,16\%), descrita Equação 7 que demonstra as variáveis utilizadas no modelo. Evidencia-se que a classe de $50 \mathrm{~m}$ foi a que mais influenciou, indicando que quanto menor a distância de extração menor será o custo de produção da operação.

$C P M=1,42-0,41 d_{50}-0,25 d_{100}-0,40 c_{1}$

\section{CONCLUSÕES}

Dentre os elementos do ciclo operacional, os deslocamentos do grapple skidder representam em média $77 \%$ do tempo total efetivo da operação de extração de árvores de Eucalyptus platyphylla.

A produtividade por hora efetiva do grapple skidder foi influenciada pela distância de extração e pela inclinação da superfície do terreno. 
O gasto monetário com combustível foi o componente de custo variável que exerceu maior impacto sobre o custo por hora programada da máquina, como resultado, influencia de modo direto os custos de produção da operação.

O custo de produção da operação do arraste de árvores indicam uma relação positiva com a distância do ramal de extração do grapple skidder.

\section{AGRADECIMENTOS}

O presente trabalho foi realizado com apoio da Coordenação de Aperfeiçoamento de Pessoal de Nível Superior - Brasil (CAPES) - Código de Financiamento 001.

\section{REFERÊNCIAS BIBLIOGRÁFICAS}

Alvares, C. A., Stape, J. L., Sentelhas, P. C., Gonçalves, J. L. M., \& Sparovek, G. (2014). Koppen's climate classification map for Brazil. Meteorologishe Zeitschrift, 22(6), 711-728. http://dx.doi.org/10.1127/0941-2948/2013/0507.

Banco Central do Brasil - BCB. (2019). Conversão de moedas. Recuperado em 5 de janeiro de 2019, de http://www4.bcb.gov.br/pec/conversao/conversao.asp

Behjou, K., Majnounian, B., Namiranian, M., \& Dvořák, J. (2008). Time study and skidding capacity of the wheeled skidder timberjack 450C in Caspian forests. Journal of Forest Science, 54(4), 183-188. http://dx.doi.org/10.17221/5/2008-JFS.

Birro, M. H. B., Machado, C. C., Souza, A. P., \& Minetti, L. J. (2002). Avaliação técnica e econômica da extração de madeira de eucalipto com track-skidder em região montanhosa. Revista Árvore, 26(5), 525-532. http://dx.doi.org/10.1590/S0100-67622002000500001.

Bodaghi, A. I., Nikooy, M., Naghdi, R., Venanzi, R., Latterini, F., Tavankar, F., \& Picchio, R. (2018). Ground based extraction on salvage logging in two high forests: a productivity and cost analysis. Forests, 9(12), 1-18.

Diggle, P. J., Heagerty, P., Liang, K. Y., \& Zeger, S. L. (2002). Analysis of longitudinal data (2nd ed.). Oxford: Oxford Science Publications.

Diniz, C. C. C., Nakajima, N. Y., Robert, R. C. G., Dolácio, C. J. F., \& Silva, F. A. (2018). Desempenho de um feller buncher em extrema variação da declividade do terreno. Advances in Forestry Science, 5(3), 381-384.

Duka, A., Porsinsky, T., Pentek, T., Pandur, Z., Vusic, D., \& Papa, I. (2018). Mobility range of a cable skidder for timber extraction on sloped terrain. Forests, 9(9), 1-11. http://dx.doi.org/10.3390/f9090526.

Fernandes, H. C., Lopes, S. E., Teixeira, M. M., Minette, L. J., Rinaldi, P. C. N., \& Bernardes, A. M. (2009). Avaliação das características técnica e econômica de um sistema de colheita florestal de árvores inteiras. Scientia Forestalis, 37(83), 225-232.

Fiedler, N. C., Rocha, E. B., \& Lopes, E. S. (2008). Análise da produtividade de um sistema de colheita de árvores inteiras no norte do Estado de Goiás. Floresta, 38(4), 577-586. http://dx.doi.org/10.5380/rf.v38i4.13153.

Food and Agriculture Organization of the United Nations - FAO. (1992). Cost control in forest harvesting and road construction (Forestry Paper, No. 99). Rome: FAO.

Ghaffariyan, M. R., Brown, M., Acuna, M., \& Kellogg, L. (2012). Productivity of roadside processing system in Western Australia. Silva Balcanica, 13, 49-60.

Hiesl, P., \& Benjamin, J. G. (2015). Can technology help improve grapple skidder and stroke delimber interactions? A simulation approach. International Journal of Forest Engineering, 26(3), 171-184. http://dx.doi.org/10.1080/14942119.2015.1108038.

Hiesl, P., Waring, T. M., \& Benjamin, J. G. (2015). The effect of hardwood component on grapple skidder and stroke delimber idle time and productivity: an agent based model. Computers and Electronics in Agriculture, 118, 270-280. http://dx.doi.org/10.1016/j.compag.2015.09.010.

Leonello, E. C., Gonçalves, S. P., \& Fenner, P. T. (2012). Efeito do tempo de experiência de operadores de harvester no rendimento operacional. Revista Árvore, 36(6), 1129-1133. http://dx.doi.org/10.1590/S0100-67622012000600013.

Lima, J. S. S., \& Leite, A. M. P. (2014). Mecanização. In C. C. Machado (Ed.), Colheita florestal (3. ed., Cap. 2 , pp. 46-73). Viçosa: Editora UFV. 
Lopes, E. S., \& Diniz, C. C. C. (2015). Produtividade do trator florestal chocker skidder na extração de madeira em terrenos declivosos. Floresta, 45(3), 625-634. http://dx.doi.org/10.5380/rf.v45i3.36409.

Lopes, E. S., Oliveira, D., \& Sampietro, J. A. (2014). Influence of wheeled types of a skidder on productivity and cost of the forest harvesting. Floresta, 44(1), 53-62. http://dx.doi.org/10.5380/rf.v44i1.31356.

Lopes, S. E., Fernandes, H. C., Minette, L. J., Silveira, J. C. M., \& Rinaldi, P. C. N. (2009). Avaliação técnica e econômica de um skidder operando em diferentes produtividades e distâncias de extração. Ciência e Agrotecnologia, 33(6), 1621-1626. http://dx.doi.org/10.1590/\$1413-70542009000600023.

Machado, C. C. (2014). Colheita florestal (3. ed.). Viçosa: Editora UFV.

Magagnotti, N., \& Spinelli, R. (2012). Good practice guidelines for biomass production studies (p. 54). Sesto Fiorentino: CNR Ivalsa.

Minette, L. J., Moreira, F. M. T., Souza, A. P., Machado, C. C., \& Silva, K. R. (2004). Análise técnica e econômica do forwarder em três subsistemas de colheita em florestas de eucalipto. Revista Árvore 28(1), 91-97. http://dx.doi.org/10.1590/S0100-67622004000100012.

Minette, L. J., Silva, E. N., Freitas, K. E., Souza, A. P., \& Silva, E. P. (2008). Análise técnica e econômica da colheita florestal mecanizada em Niquelândia, Goiás. Revista Brasileira de Engenharia Agrícola e Ambiental, 12(6), 659-665. http://dx.doi.org/10.1590/S1415-43662008000600014.

Moreira, F. M. T., Souza, A. P., Machado, C. C., Minetti, L. J., \& Silva, K. R. (2004). Avaliação operacional e econômica do feller buncher em dois subsistemas de colheita de florestas de eucalipto. Revista Árvore, 28(2), 199-205. http://dx.doi.org/10.1590/S0100-67622004000200006.

Moskalik, T., Borz, S. A., Dvorak, J., Ferencik, M., Glushkov, S., Muiste, P., Lazdins, A., \& Styranivsky, O. (2017). Timber harvesting methods in Eastern European countries: a review. Croatian Journal of Forest Engineering, 38, 231-241.

Mousavi, R. (2012). Time consumption, productivity, and cost analysis of skidding in the Hyrcanian forest in Iran. Journal of Forestry Research, 23(4), 691-697. http://dx.doi.org/10.1007/s11676-0120265-1.

Nelder, J. A., \& Wedderburn, R. W. (1972). Generalized linear models. Journal of the Royal Statistical Society. Series A (General), 135(3), 370-384. http://dx.doi.org/10.2307/2344614.

Oliveira, R. J., Machado, C. C., Fernandes, H. C., \& Canto, J. L. (2008). Clambunk skidder: avaliação econômica no arraste de madeira. Semina: Ciências Agrárias, 29(3), 547-556. http://dx.doi.org/10.5433/1679-0359.2008v29n3p547.

Ovaskainen, H., Uusitalo, J., \& Väätäinen, K. (2004). Characteristics and significance of a harvester operators' working technique in thinnings. International Journal of Forest Engineering, 15(2), 36-41. http://dx.doi.org/10.1080/14942119.2004.10702498.

Pereira, A. L. N., Lopes, E. S., \& Dias, A. N. (2015). Análise técnica e de custo do feller buncher e skidder na colheita de madeira em diferentes produtividades do povoamento. Ciência Florestal, 25(4), 981 989. http://dx.doi.org/10.5902/1980509820659.

Proto, A. R., Macri, G., Visser, R., Russo, D., \& Zimbalatti, G. (2018). Comparison of timber extraction productivity between winch and grapple skidding: a case study in Southern Italian forests. Forests, 9(2), 1-12. http://dx.doi.org/10.3390/f9020061.

Rocha, E. B., Fiedler, N. C., Alves, R. T., Lopes, E. S., Guimarães, P. P., \& Peroni, L. (2009). Produtividade e custos de um sistema de colheita de árvores inteiras. Cerne, 15(3), 372-381.

Santos, H. G., Almeida, J. A., Oliveira, J. B., Lumbreras, J. F., Anjos, L. H. C., Coelho, M. R., Jacomine, P. K. T., Cunha, T. J. F., \& Oliveira, V. A. (2018). Sistema brasileiro de classificação de solos. 5. ed. Brasília: Editora EMBRAPA.

Santos, P. H. A., Souza, A. P., Marzano, F. L. C., \& Minette, L. J. (2013). Produtividade e custos de extração de madeira de eucalipto. Revista Árvore, 37(3), 511-518. http://dx.doi.org/10.1590/S010067622013000300014.

SAS Institute. (2012). Statistical analysis system for Windows. release 9.2. Cary.

Seixas, F. (2008). Extração florestal. In C. C. Machado (Ed.), Colheita florestal (2. ed., Cap. 4, pp. 97-145). Viçosa: UFV.

Seixas, F., \& Castro, G. P. (2014). Extração. In C. C. Machado (Ed.), Colheita florestal (3. ed., Cap. 4, pp. 106-161). Viçosa: UFV.

Simões, D., Fenner, P. T., \& Esperancini, M. S. T. (2014). Produtividade e custos do feller buncher e Processador Florestal em povoamentos de eucalipto de primeiro corte. Ciência Florestal, 24(3), 621 630. http://dx.doi.org/10.5902/1980509815742. 
Simões, D., Miyajima, R. H., Tonin, R. P., Fenner, P. T., \& Batistela, G. C. (2018). Incorporation of uncertainty in technical and economic analysis of a feller-buncher. Floresta, 48(3), 403-412. http://dx.doi.org/10.5380/rf.v48i3.56404.3

Souza, A., Machado, C. C., Minetti, L. J., \& Jacovine, L. A. G. (2002). Perspectivas na área de colheita e transporte florestal. Revista Madeira, (51), 52-62.

Spinelli, R., Cuchet, E., \& Roux, P. (2007). A new feller-buncher for harvesting energy wood: results from a European test programme. Biomass and Bioenergy, 31(4), 205-210. http://dx.doi.org/10.1016/j.biombioe.2006.08.002.

Stevenson, W. J. (2001). Administração das operações de produção (6. ed.). Rio de Janeiro: LTC.

Tiernan, D., Zeleke, G., Owende, P. M. O., Kanali, C. L., Lyons, J., \& Ward, S. M. (2004). Effect of working conditions on forwarder productivity in cut-to-length timber harvesting on sensitive forest sites in Ireland. Biosystems Engineering, 87(2), 167-177. http://dx.doi.org/10.1016/j.biosystemseng.2003.11.009.

Visser, R., \& Stampfer, K. (2015). Expanding ground-based harvesting onto steep terrain: a review. Croatian Journal of Forest Engineering, 36, 321-331.

Vusic, D., Susnjar, M., Marchi, E., Spina, R., Zecic, Z., \& Picchio, R. (2013). Skidding operations in thinning and shelterwood cut of mixed stands-work productivity, energy inputs and emissions. Ecological Engineering, 61, 216-223. http://dx.doi.org/10.1016/j.ecoleng.2013.09.052.

Contribuição dos Autores: RHM: conceituação, curadoria de dados, análise formal, escrita-primeira redação, escrita-revisão e edição, JRSP: investigação, metodologia, visualização, escrita-revisão e edição, PTF: conceituação, obtenção de financiamento, recursos, supervisão, validação, DS: análise formal, metodologia, escrita-primeira redação, escrita-revisão e edição. 